\title{
MiR-103a promotes tumour growth and influences glucose metabolism in hepatocellular carcinoma
}

Yuling Liu ${ }^{1,3}$, Yuanzhou Zhang ${ }^{1,3}$, Bowen Xiao ${ }^{1,3}$, Ning Tang ${ }^{1,3}$, Jingying Hu ${ }^{1}$, Shunshun Liang ${ }^{1}$, Yechun Pang ${ }^{1}$, Huili Xu ${ }^{1}$, Junping Ao ${ }^{1}$, Juan Yang ${ }^{1}$, Xiaofei Liang ${ }^{1}$, Lin Wei ${ }^{1}$, Yunfeng Wang ${ }^{2 \times}$ and Xiaoying Luo ${ }^{1{ }^{凶}}$

(c) The Author(s) 2021

Hepatocellular carcinoma (HCC) is a common and high-mortality cancer worldwide. Numerous microRNAs have crucial roles in the progression of different cancers. However, identifying the important microRNAs and the target biological function of the microRNA in HCC progression is difficult. In this study, we selected highly expressed microRNAs with different read counts as candidate microRNAs and then tested whether the microRNAs were differentially expressed in HCC tumour tissues, and we found that their expression was related to the HCC prognosis. Then, we investigated the effects of microRNAs on the cell growth and mobility of HCC using a real-time cell analyser (RTCA), colony formation assay and subcutaneous xenograft models. We further used deep-sequencing technology and bioinformatic analyses to evaluate the main functions of the microRNAs. We found that miR-103a was one of the most highly expressed microRNAs in HCC tissues and that it was upregulated in HCC tissue compared with the controls. In addition, high miR-103a expression was associated with poor patient prognosis, and its overexpression promoted HCC cell growth and mobility. A functional enrichment analysis showed that miR-103a mainly promoted glucose metabolism and inhibited cell death. We validated this analysis, and the data showed that miR-103a promoted glucose metabolism-likely function and directly inhibited cell death via ATP11A and EIF5. Therefore, our study revealed that miR-103a may act as a key mediator in HCC progression.

Cell Death and Disease (2021)12:618; https://doi.org/10.1038/s41419-021-03905-3

\section{INTRODUCTION}

Hepatocellular carcinoma (HCC) is one of the most common cancers worldwide [1] and remains the third leading cause of cancer mortality [2-5]. Although great improvements have been made in diagnostic and surgical techniques and the development of new molecularly targeted drugs, the 5-year overall survival rate is still low [4]. In addition, some patients progress to advanced stages of the disease due to recurrence and metastasis $[6,7]$. Therefore, it is necessary to gain a full understanding of the molecular mechanisms underlying the tumorigenesis and tumour progression of HCC.

MicroRNAs (miRNAs/miRs) are a class of small noncoding RNA molecules that are $\sim 22$ nucleotides in length [8-10] and regulate gene expression and cellular processes by targeting messenger RNA (mRNA) transcripts [11]. MicroRNAs take part in many important biological processes, including early embryonic development [12] and fat metabolism [13], and they even regulate the differentiation of stem cells [14]. However, the abnormal expression of miRNAs is closely related to the occurrence and development of human cancer (cell proliferation [15], apoptosis [16], and cell death [17]). MicroRNAs have been shown to play crucial roles in the development and progression of different kinds of cancer, including HCC [18-20]. For example, miR-29c-3p acts as a tumour suppressor in HCC by targeting DNMT3B and the LATS1-associated Hippo signalling pathway [21]. MiR-541 potentiates the response of human HCC to sorafenib treatment by inhibiting autophagy [22]. A recent study also indicated that miR-1269b downregulated SVEP1 expression and promoted HCC proliferation and metastasis, likely through the PI3K/Akt signalling pathway [23]. In addition, previous studies have shown that many abnormally expressed microRNAs are closely associated with the prognosis of HCC patients $[6,24,25]$. However, these analyses are mainly based on the results of the differential expression between tumour tissues and normal tissues, whereas the expression level of microRNA is less frequently considered.

The rapid development of high-throughput sequencing technologies and comprehensive bioinformatics analyses allows for the analysis of important differentially regulated molecules in HCC progression and their major functions [26]. Although many studies have focused on differential molecular regulation in HCC progression and its function [27, 28], the main regulatory factors or the main nodes in the regulatory network remain issues that need to be considered. To address this challenge, we analysed HCC microRNA expression data acquired from The Cancer Genome Atlas (TCGA) database. We screened for differentially expressed microRNAs (DEMs) in tumours vs adjacent normal tissues based on the expression level of microRNAs. We hypothesize that these high-expression DEMs have significant

\footnotetext{
${ }^{1}$ State Key Laboratory of Oncogenes and Related Genes, Shanghai Cancer Institute, Renji Hospital, Shanghai Jiao Tong University School of Medicine, Shanghai 200032, People's Republic of China. ${ }^{2}$ Department of General Surgery, Pudong New Area People's Hospital, Shanghai University of Medicine \& Health Sciences, Shanghai, China. ${ }^{3}$ These authors

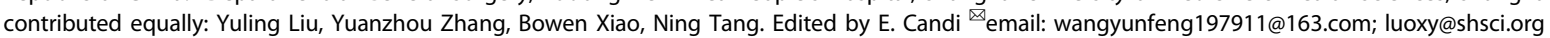

Received: 28 February 2021 Revised: 3 June 2021 Accepted: 7 June 2021

Published online: 15 June 2021 
functions in HCC progression, and investigations of these highexpression DEMS may obtain information about the main factors or nodes in the regulatory network for HCC progression.

In the present study, we found that miR-103a is a highly expressed and upregulated microRNA in HCC tissues and showed that its high expression is associated with poor prognosis in patients with HCC. We further found that miR-103 promotes cell proliferation, migration, invasion and glucose metabolism in vitro and that miR-103a promotes tumour growth in vivo. Glucose metabolism is the main function of miR-103a. Our study suggested that high expression and upregulation of miR-103a are the main differentiation factors in the regulatory network and indicated that the ability of miR-103a to promote glucose metabolism function might represent the main change in HCC progression.

\section{RESULTS \\ miR-103a is a highly expressed and upregulated microRNA in HCC tissues}

We performed an expression analysis of publicly available microRNA sequencing data from HCC patients in the TCGA database. Then, microRNAs were sorted from high to low according to the read counts in HCC tissues. We identified 15 top-ranked microRNAs (Fig. 1A), and 6 were upregulated while 9 were downregulated (Fig. 1A, according to the read counts, not normalized). Furthermore, we detected the microRNA proportion compared to the total microRNA sequencing read counts in HCC tissues, and the data showed that the miR-103a read counts were $1.458 \%$ of the total microRNA read counts (Fig. 1B). The above data showed that miR-103a was a high-expression microRNA. miR103a plays a vital role in physiological [29] and pathological processes [30]. Because of the limited studies of liver cancer, we chose this microRNA as a candidate.

High-expression/differentiated expression microRNAs might play an important role in HCC progression. To validate that miR$103 a$ is a DEM in HCC, we checked the miR-103a expression in TCGA datasets after normalizing the read counts. The data showed that miR-103a expression was 1.6-fold upregulated in HCC tissues $(n=372)$ compared with normal adjacent tissues $(n=50)$ (Fig. 1C, $p<0.0001$, unpaired). High expression of miR-103a was associated with poor prognosis of HCC patients in TCGA (Fig. 1D, follow-up cut-off is 36 months).

Alternatively, miR-103a levels in 89 primary HCC and paired adjacent tissue samples were evaluated by real-time PCR (Table S1)Then, we checked the expression of miR-103a in our cohort, and the data showed that miR-103a was 1.94-fold upregulated in HCC tissues compared with adjacent tissues (Fig. $2 \mathrm{~A}, p<0.0001$, paired $t$ test, $n=89$ ). To evaluate the potential prognostic value of miR-103a in HCC, we performed an overall survival analysis of our cohort, and the data showed that the upregulation of miR-103a in HCC patients was correlated with poorer survival (Fig. 2B, $p=0.0447$ ). As expected, we obtained similar results in our cohort as the TCGA database analysis.

These results showed that miR-103a is a highly expressed and upregulated microRNA in HCC tissues, and its high expression is associated with poor prognosis.

\section{miR-103a promotes the proliferation of HCC cells in vitro}

To further investigate the biological function of miR-103a in HCC, four types of HCC cells (SMMC-7721, MHCC97L, MHCC97H and HCCLM3), which are widely used in the study of HCC, were transfected with lentivirus-miR-103a or lentivirus-control, and the transfection efficiency was determined via qRT-PCR analysis (Fig. 2C-E).

To identify the potential function of miR-103a, a real-time cell analyzer (RTCA) was performed to measure the proliferation rates of miR-103a-overexpressing HCC cells. miR-103a overexpression promoted cell proliferation (Fig. $3 \mathrm{~A}, p<0.0001$ ). Furthermore, the colony formation assay results suggested that miR-103a overexpression remarkably promoted cell colony formation abilities compared with the negative control groups (Fig. 3B-E), and downregulated miR-103 expression in SMMC-7721 inhibited cell proliferation (Fig. S1B).

These data showed that miR-103a promotes cell growth in vitro.

miR-103a promotes HCC cell migration and invasion in vitro Next, we performed cell migration assays and invasion assays to study the influence of miR-103a on HCC mobility. The data showed that miR-103a overexpression obviously promoted HCC cell migration and invasion relative to the negative control groups (Fig. 4A, B). And downregulated miR-103 expression in SMMC-7721 inhibited cell invasion (Fig. S1C). These data showed that miR-103a overexpression promotes the mobility of HCC cells.

\section{miR-103a influences glucose metabolism in HCC cells}

To obtain a more comprehensive functional analysis of miR-103, we checked the microRNA expression profile of miR-103aoverexpressing MHCC97L cells by next-generation sequencing. We analyzed the sequencing data by STAR-htseq workflows and then obtained the gene functional enrichment information by the EGSEA r package. In the biological function analysis, we found that the glucose metabolic process, cell death and glycolysis were the top three biological functions (Fig. 5A), with 14 genes participating in these biological functions (Fig. 5B).

To validate the gene functional enrichment information, we assessed the effect of miR-103a overexpression on glycolytic metabolism via a seahorse assay. The results showed that the overexpression of miR-103a enhanced the extracellular acidification rate (ECAR), which reflects the overall glycolytic flux, and decreased the $O C R$, which is an indicator of mitochondrial respiration (Fig. 5C, D). And downregulated miR-103 expression in SMMC-7721 decreased the ECAR and enhanced the OCR (Fig. S1D/E).

These results revealed that miR-103a mainly influences glucose metabolism in HCC.

\section{ATP11A and EIF5 are direct targets of miR-103a}

MicroRNAs can repress transcription by binding to complementary sequences in the $3^{\prime}$-UTRs. A target prediction analysis (miRDB) was performed to explore the potential direct targets of miR-103a, and the findings were combined with the results for the downregulated genes in miR-103a-overexpressing MHCC97L cells. Of the 6 candidate genes, ATP11A and EIF5 (Fig. 6A) could interact with the gene enrichment functional network (STRINGdb). Furthermore, the $3^{\prime}$-UTR of ATP11A contains five putative complementary binding sites for miR-103a, and the $3^{\prime}$-UTR of EIF5 contains two putative complementary binding sites for miR103a (Fig. 6B). To verify that ATP11A and EIF5 are targets of miR103a, HEK293T cells were transfected with miR-103a agomiR and the luciferase reporter vector harbouring the wild-type or mutant 3'-UTR of ATP11A and EIF5, respectively. The results demonstrated that overexpression of miR-103a significantly decreased the luciferase activity of cells expressing the wild-type but not the mutant 3'-UTR of ATP11A and EIF5 (Fig. 6C). In addition, miR-103a antagomiRs enhanced the mRNA expression of ATP11A and EIF5 in SMMC-7721 cells. In contrast, miR-103a agomiR transfection had the opposite effect on the mRNA of ATP11A and EIF5 (Fig. 6D). Moreover, an analysis using the TCGA database demonstrated that ATP11A and EIF5 had significant negative correlations with miR-103a (ATP11A: $p=0.0348, r=-0.1149$; ElF5: $p=0.0256$, $r=-0.1229$; Fig. 6E).

Together, these findings revealed that miR-103a could regulate glucose metabolic processes, cell death and glycolysis by directly targeting ATP11A and EIF5. 
A

miR-21

miR-22

miR-148a miR-143

miR-192
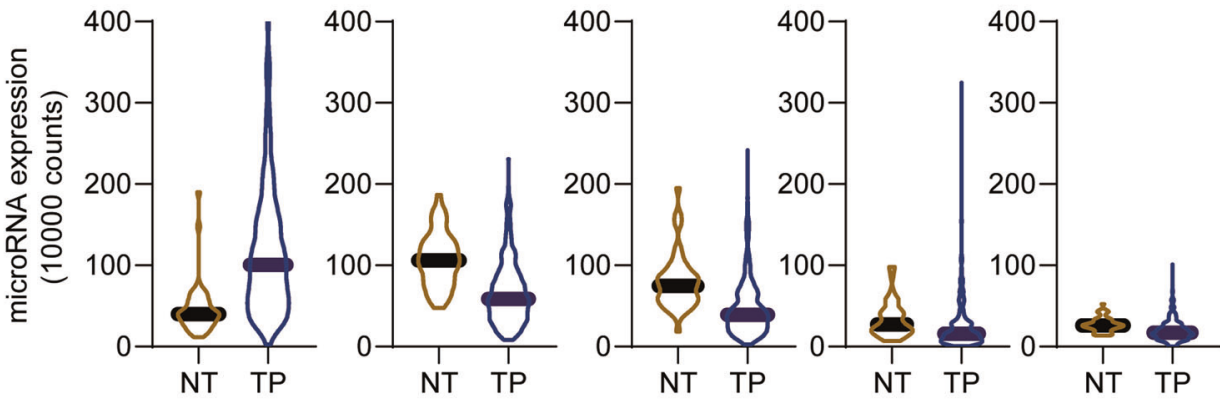

miR-30a

miR-122

miR-10b

miR-30e

miR-30d
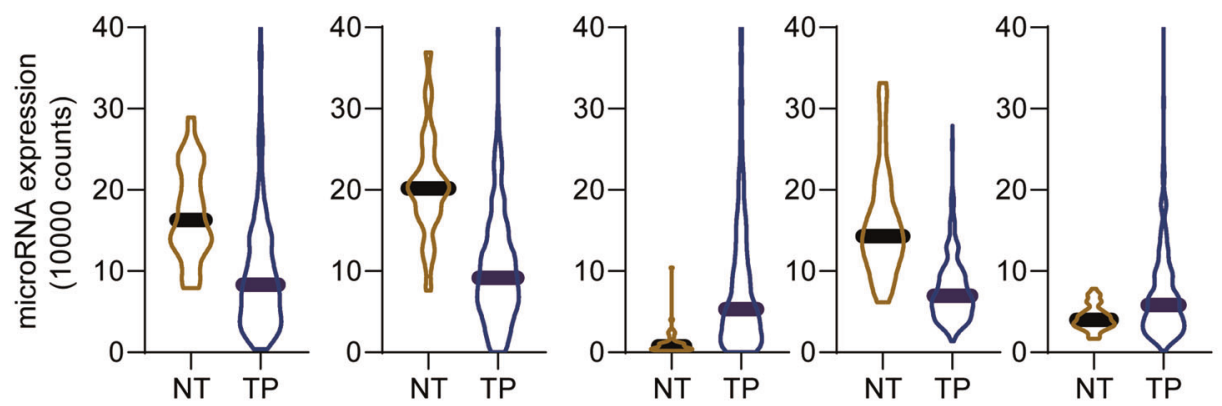

miR-10a

miR-103a-2

miR-103a

miR-99b

miR-92a
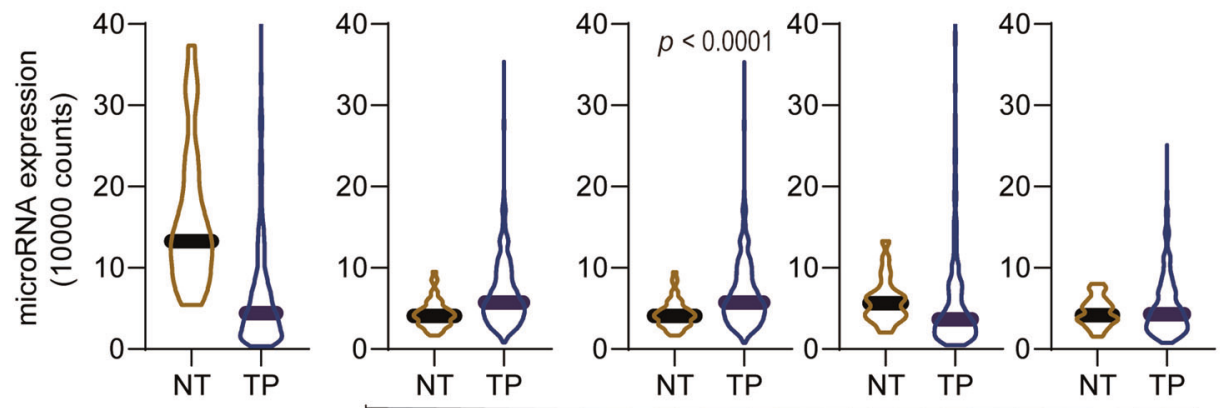

B

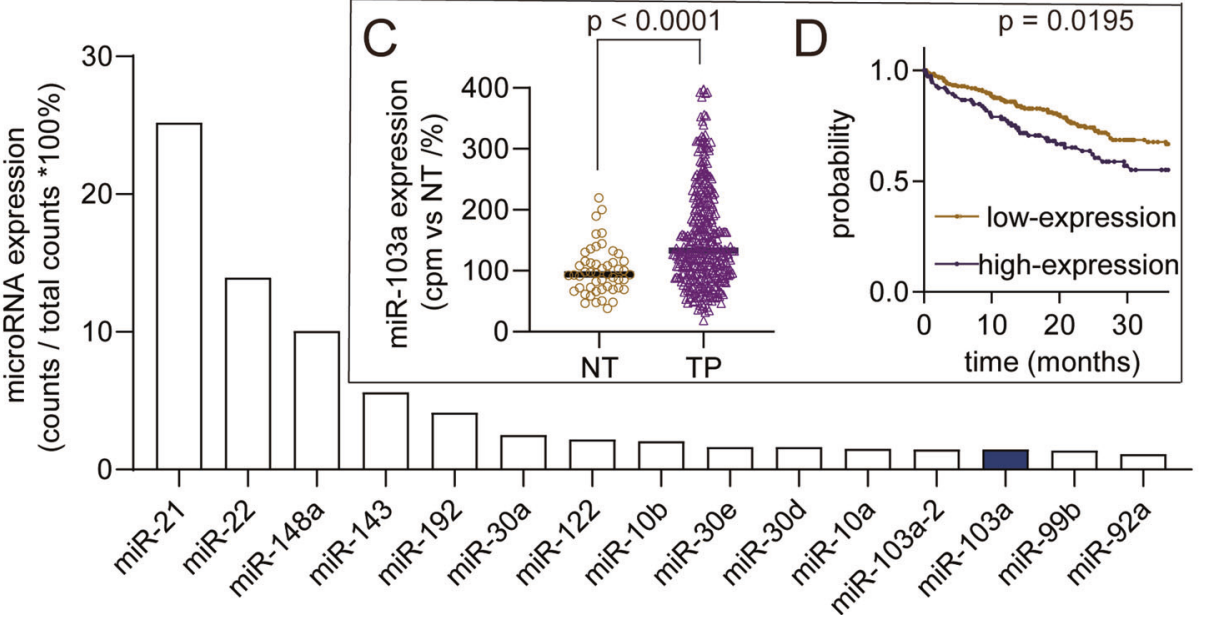

Fig. 1 miR-103a is a highly expressed/upregulated microRNA in HCC, and its high expression is related to poor prognosis in TCGA datasets. A Fifteen highest microRNA read counts in hepatocellular carcinoma tissues $(n=372)$ and adjacent normal tissues $(n=50)$. B MiR103 a reads in next-generation sequencing accounted for $1.458 \%$ of the total reads, and miR-103a was the 13 th most highly expressed microRNA in HCC tissues. C miR-103a expression levels (normalized reads) were upregulated in HCC tissues $(n=372)$ compared with adjacent normal tissues $(n=50)$. D Kaplan-Meier analysis showing that the level of miR-103a was negatively correlated with the overall survival rate of HCC patients (follow-up threshold was 36 months). NT adjacent tumour tissues, TP tumour tissues. miR-103a microRNA-103a, HCC hepatocellular carcinoma cells. These data were obtained from TCGA database. 
A

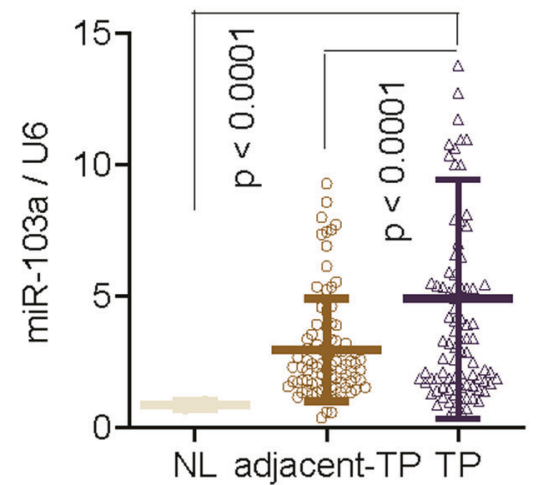

B

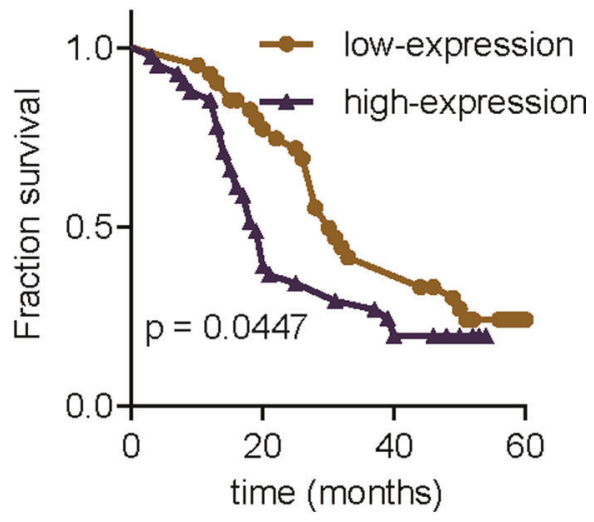

C

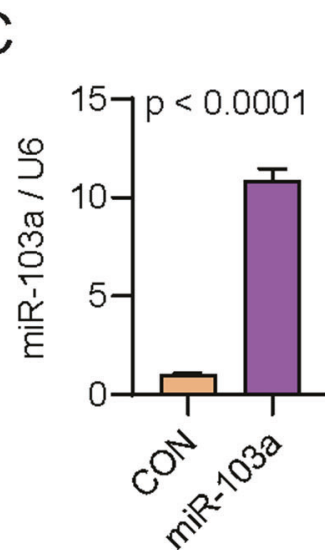

D

E
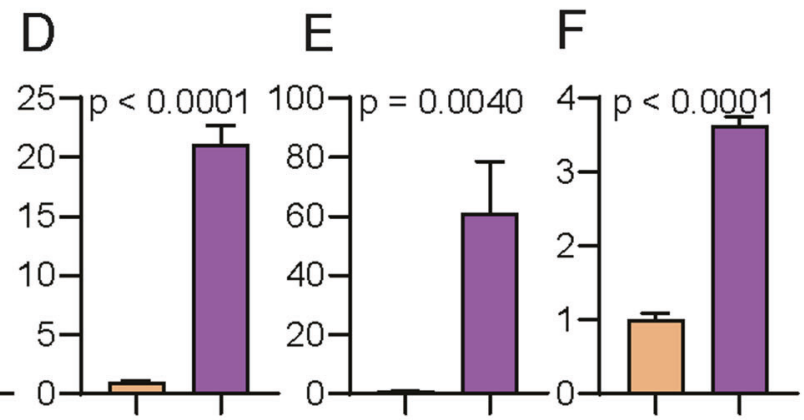

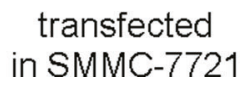

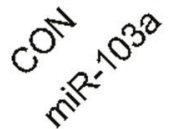

transfected

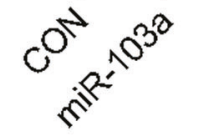

transfected

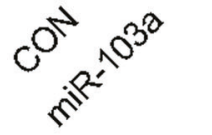

transfected

in $\mathrm{MHCC} 97 \mathrm{H}$

in HCCLM3

Fig. 2 miR-103a expression was upregulated in HCC and related to poor prognosis in the Renji cohort. A Reverse transcriptionquantitative PCR analysis was performed to assess miR-103a expression in 89 paired HCC tissues and adjacent normal tissues and two healthy volunteer liver tissues. B Survival curves suggested that increased miR-103a expression was significantly associated with unfavourable prognosis of patients with HCC. C-F Reverse transcription-quantitative PCR analysis was performed to validate miR-103a expression in SMMC$7721(p<0.001)$, MHCC97H $(p<0.001)$, MHCC97L $(p<0.01)$ and HCCLM3 $(p<0.001)$ cells transfected with miR-103a lentivirus. All results are representative of at least three independent experiments.

\section{Overexpression of miR-103a promotes HCC growth in a xenograft model}

To further confirm the in vitro findings, we observed the biological roles of miR-103a in vivo. miR-103a overexpression in SMMC-7721 cells increased the tumour volume 2.79 -fold relative to that of its control (Fig. 7A) and increased the tumour weight 3.36-fold relative to its control (Fig. 7B), and the representative images are shown in Fig. 7C. MiR-103a overexpression in MHCC97L cells increased the tumour volume 2.35 -fold relative to that of the control (Fig. 7D) and increased the tumour weight 1.82-fold relative to that of the control (Fig. 7E), and the representative images are shown in Fig. 7F.

The results demonstrated that after implantation of miR-103aoverexpressing cells, tumour xenografts grew significantly faster than those in the control group.

\section{DISCUSSION}

In this study, we found that miR-103a was a highly expressed and upregulated microRNA in HCC tissues from TCGA. In addition, the high expression of miR-103a was correlated with poor prognosis in HCC patients. Functional experiments revealed that miR-103a overexpression promoted HCC cell growth, migration, invasion and glucose metabolism. Furthermore, the results of bioinformatics methods and dual-luciferase reporter assays showed that miR-103a mainly affected glucose metabolism and cell death by targeting ATP11A and EIF5 directly, thus contributing to HCC cell growth and mobility.

Previous studies have shown that miR-103a plays a critical role in the progression of cancer metastasis. miR-103/107 attenuates miRNA biosynthesis by targeting Dicer, induces the epithelial-tomesenchymal transition (EMT), and promotes breast cancer cell metastasis [30]. miR-103/107-mediated repression of DAPK and KLF4 promoted metastasis in CRC [31]. miR-103a plays both an oncogenic and tumour-suppressive role in various types of cancers. For example, miR-103 promotes proliferation and metastasis by targeting KLF4 in gastric cancer [32] and regulates triple-negative breast cancer cell migration and invasion by targeting olfactomdin4 [33], while miR-103a functions as a tumour suppressor by directly targeting programmed cell death 10 in NSCLC [34]. A recent study showed that upregulation of serum miR-103 predicts an unfavourable prognosis in patients with colorectal cancer [35]. Taken together, these results suggest that promoting cancer metastasis might be the main function of miR-103a.

miR-103a is also involved in cell growth and metastasis in liver cancer. Previous reports indicated that miR-103a is upregulated in HCC. Moreover, miR-103 promotes HCC cell proliferation and migration in the simulation transition zone of RFA through the 
A

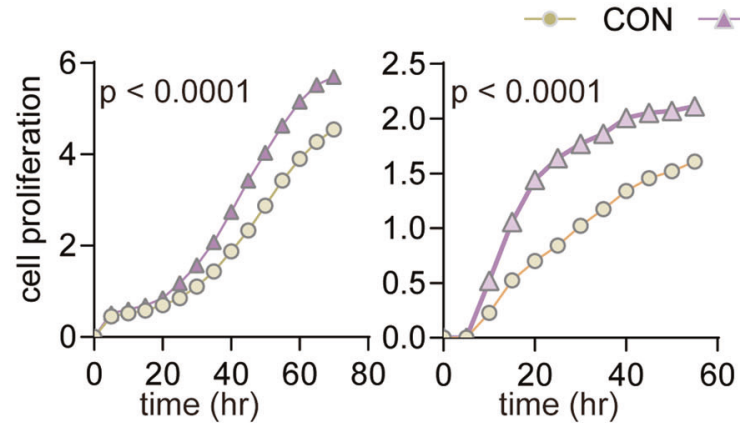

miR-103a
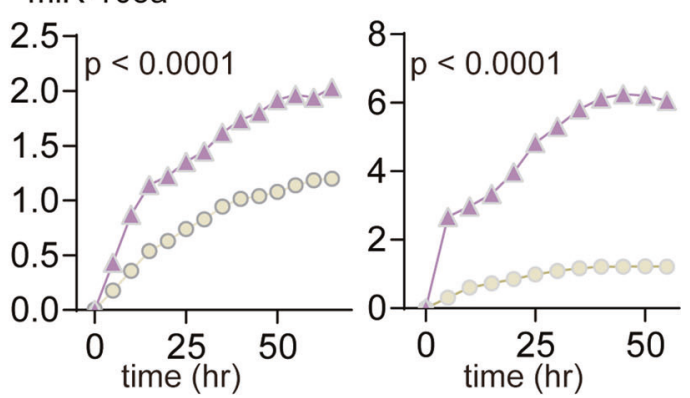

B

SMMC-7721
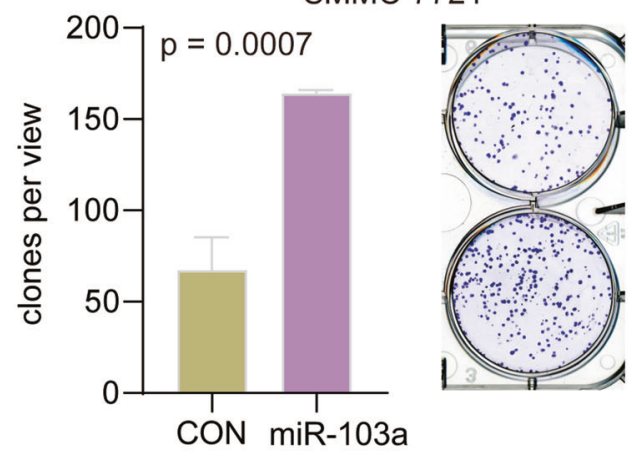

C

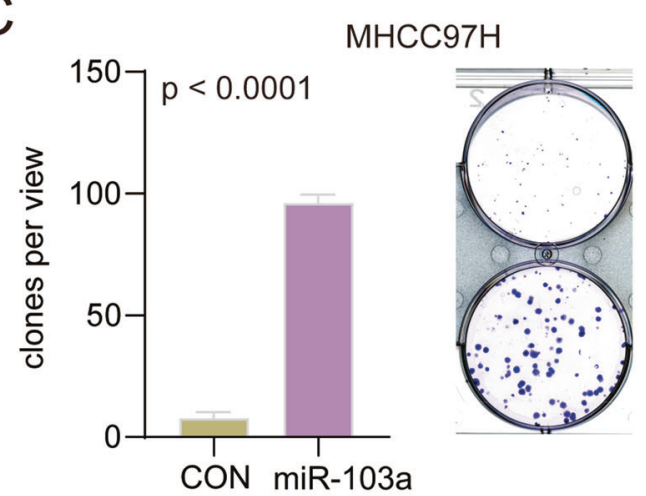

D

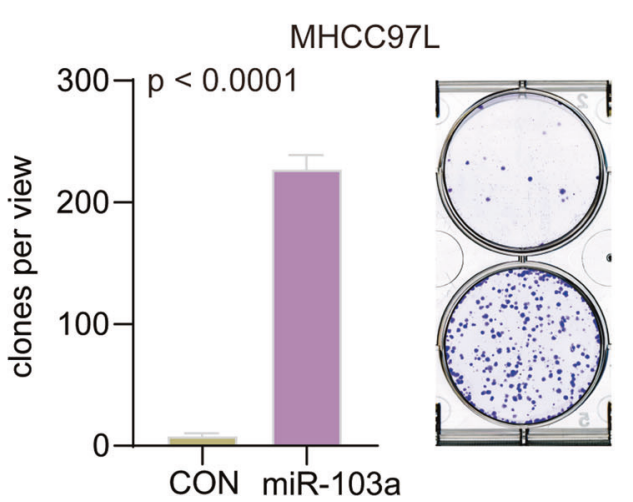

E

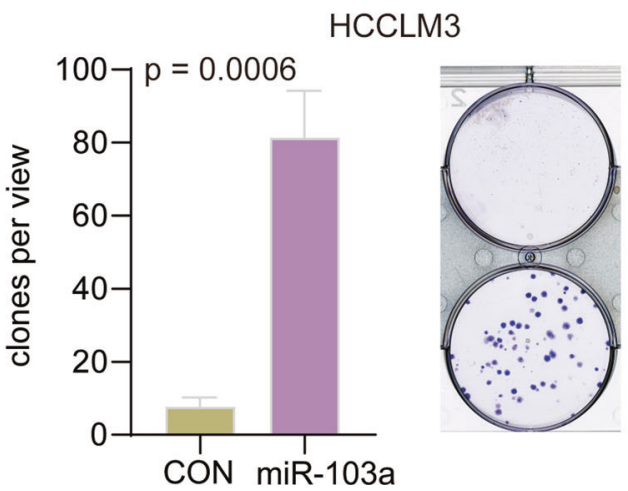

Fig. 3 miR-103a overexpression promoted hepatocellular carcinoma cell growth. A RTCA was performed to determine the proliferation of SMMC-7721, MHCC97H, MHCC97L and HCCLM3 cells overexpressing miR-103a. B-E Colony formation assay was performed to examine the colony formation abilities of SMMC-7721, MHCC97H, MHCC97L and HCCLM3 cells overexpressing miR-103a. ${ }^{*} p<0.5,{ }^{* *} p<0.01,{ }^{* * *} p<0.001$. All results are representative of at least three independent experiments.

PI3K/Akt signalling pathway by targeting PTEN [36], promotes the metastasis and EMT of HCC by directly inhibiting LATS2 [37], and regulates HCC growth by targeting AKAP12 [38]. Moreover, hepatoma cell-secreted exosomal microRNA-103 has been shown to increase vascular permeability and promote metastasis by targeting junction proteins [39]. However, the above studies mainly focused on exploring the effects and mechanisms of miR103a on HCC cell growth without addressing the role of miR-103a in glucose metabolism in $\mathrm{HCC}$ or determining its main function.

In our study, the bioinformatics analysis showed that the EMT, which is the main malignant biological behaviour of miR-103a in the literature, was the fifth most important biological process. Interestingly, miR-103a was mainly involved in the regulation of glucose metabolism and cell death. In the current study, we revealed a novel function of miR-103a in regulating the glucose metabolism phenotypes of HCC.
The liver is a major metabolic organ and a major site of glucose metabolism [40,41], which is an important process in cancer cell growth [42]. Changes in glucose metabolism have been listed as an important factor that directly contributes to carcinogenesis $[43,44]$. However, to the best of our knowledge, no causal association has been established between miR-103a and glucose metabolism in HCC.

In summary, we identified miR-103a as one of the most highly expressed microRNAs in HCC tissues, found that it was upregulated in HCC tissues compared with the controls, and showed that high expression of miR-103a was associated with poor patient prognosis. Moreover, miR-103a overexpression promoted HCC cell growth and mobility, and the functional enrichment analysis showed that miR-103a mainly promoted glucose metabolism and inhibited cell death. We validated this analysis, and the results showed that miR103a directly influenced glucose metabolism and inhibited cell death 


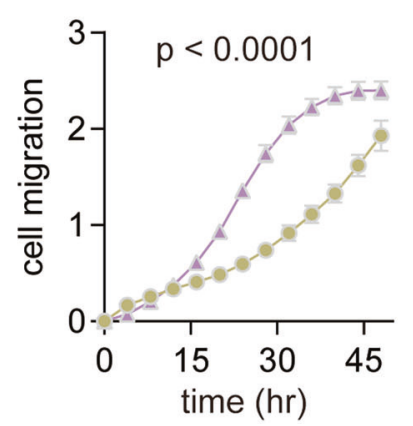

B

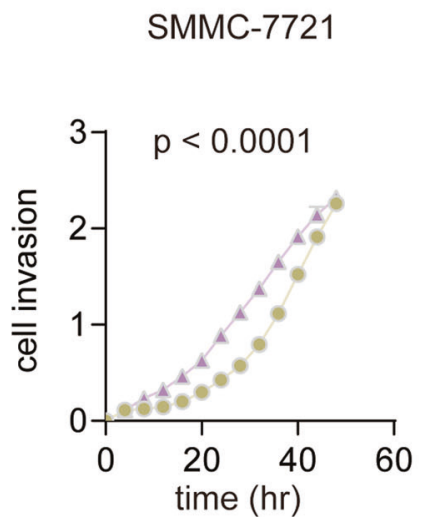

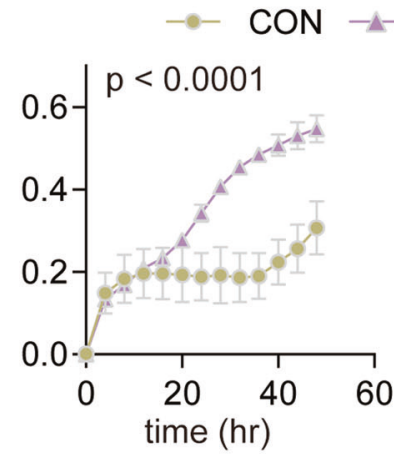

$\mathrm{MHCC} 97 \mathrm{H}$

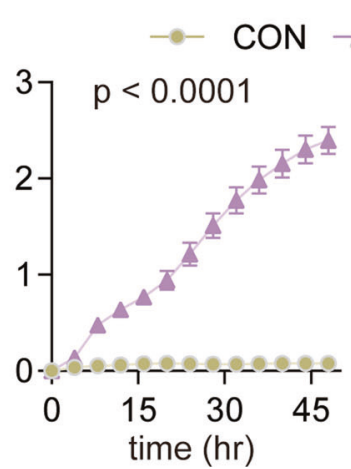

miR-103a
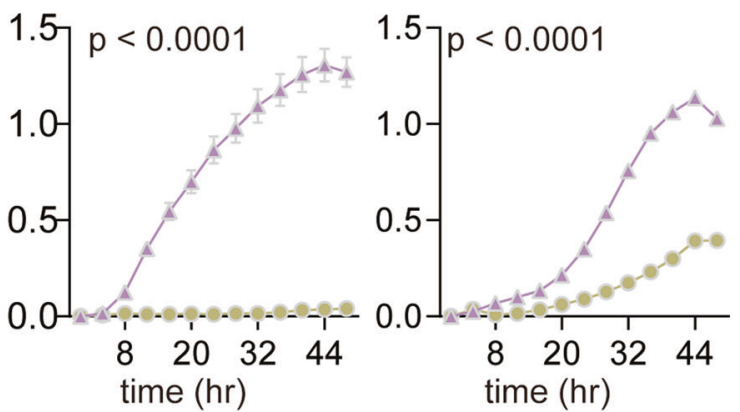

MHCC97L

HCCLM3

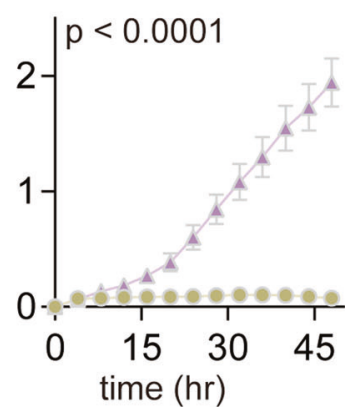

Fig. 4 miR-103a promoted the mobility of hepatocellular carcinoma cells. A Overexpression of miR-103a promotes HCC cell migration. The migration ability of SMMC-7721, MHCC97H, MHCC97L and HCCLM3 cells overexpressing miR-103a was detected by RTCA. B Overexpression of miR-103a promoted HCC cell invasion. The invasion ability of SMMC-7721, MHCC97H, MHCC97L and HCCLM3 cells overexpressing miR-103a was detected by RTCA. All results are representative of at least three independent experiments.

via ATP11A and EIF5. Therefore, our study revealed that miR-103a may act as a key mediator in HCC progression.

\section{MATERIALS AND METHODS}

\section{Cell lines and culture}

Four human HCC cell lines (SMMC-7721, MHCC97H, MHCC97L and HCCLM3) and HEK293T cells were purchased from The Cell Bank of Type Culture Collection of the Chinese Academy of Sciences (Shanghai, China). All cell lines were cultured in Dulbecco's modified Eagle's medium (DMEM; Gibco, USA) supplemented with $10 \%$ foetal bovine serum (FBS; Gibco, USA), 100 units $/ \mathrm{mL}$ penicillin and $100 \mu \mathrm{g} / \mathrm{mL}$ streptomycin (Gibco, USA) and incubated at $37{ }^{\circ} \mathrm{C}$ in humidified incubators containing $5 \% \mathrm{CO}_{2}$.

\section{Clinical samples and TCGA data analysis}

Eighty-nine paired samples of tumour tissues (TPS) and their corresponding nontumour tissues (NTs) from patients with HCC and two normal liver tissues were obtained from the surgical specimen archives of Guangxi Medical University, Guangxi Province, China. One of the two normal liver tissue samples was collected from a person who died because of an accident; the other was purchased from Clontech (Palo Alto, CA). The whole transcriptome sequencing (RNA-seq) data of 374 liver TPs and 50 adjacent NTs were obtained from TCGA liver cancer dataset (LIHC) (http:// cancergenome.nih.gov). Informed consent was obtained for all human materials, and the institutional ethics review committee of the Shanghai Cancer Institute approved the protocols used in this study.

\section{RNA extraction and quantitative real-time polymerase chain reaction analysis}

Total RNA was extracted from cell lines and tissue samples using a TRlzol kit (Invitrogen, Carlsbad, CA, USA). RNA $(1 \mu \mathrm{g})$ was reverse-transcribed into cDNA immediately using a Prime-Script RT kit (Takara, Shiga, Japan) following the manufacturer's instructions. QRT-PCR was carried out with SYBR Premix EX Tag (Takara) on an ABI Prism 7500 fast RT-PCR instrument (Applied Biosystems, Foster City, CA). Each experiment was performed in triplicate. MicroRNA qRT-PCR primers were obtained from RiboBio (Guangzhou, China). The microRNA expression levels were calculated using the delta-delta Ct method, with RNU6B as an internal control. A Ct value of 35 was set as the cut-off value for a nondetected classification. $\beta$-actin was used as the internal reference gene for gene expression. Objective CT values were normalized to $\beta$-actin, and the $2^{-\Delta \Delta \mathrm{Ct}}$ method was used to calculate relative mRNA levels of gene expression. The primer sequences contained in this study were as follows (http://pga.mgh.harvard. edu/primerbank/):

ATP11A: F, 5'-TACCCAGACAACAGGATCGTC- $3^{\prime}$ and

R, 5'- AGCCGTCACAGTAATGACAAAG-3';

EIF5: F, 5'- AGCGTGTCAGACCAGTTCTAT-3' and

R, 5' - CTGTCTTGATTCCATTGCCTTTG-3';

$\beta$-actin: F, 5'-TTGTTACAGGAAGTCCCTTGCC- $3^{\prime}$ and

$\mathrm{R}, 5^{\prime}$ - ATGCTATCACCTCCCCTGTGTG-3'.

\section{Lentiviral production and transduction}

Particles carrying the hsa-pri-miR-103a precursor and its control were purchased from GENECHEM (Shanghai, China). Lentiviral transduction was carried out according to advice from GENECHEM. The high expression was validated by qRT-PCR.

\section{Proliferation, colony formation, migration and invasion analysis}

Cell proliferation, invasion and migration assays were measured with the xCELLigence System's Real-Time Cell Analyzer (RTCA, Roche/ACEA Biosciences) placed in a humidified incubator and maintained at $37^{\circ} \mathrm{C}$ with $95 \%$ air $/ 5 \% \mathrm{CO}_{2}$. This system continuously monitored electrical impedance created by cell adhesion and proliferation in a microelectrode-integrated 
A

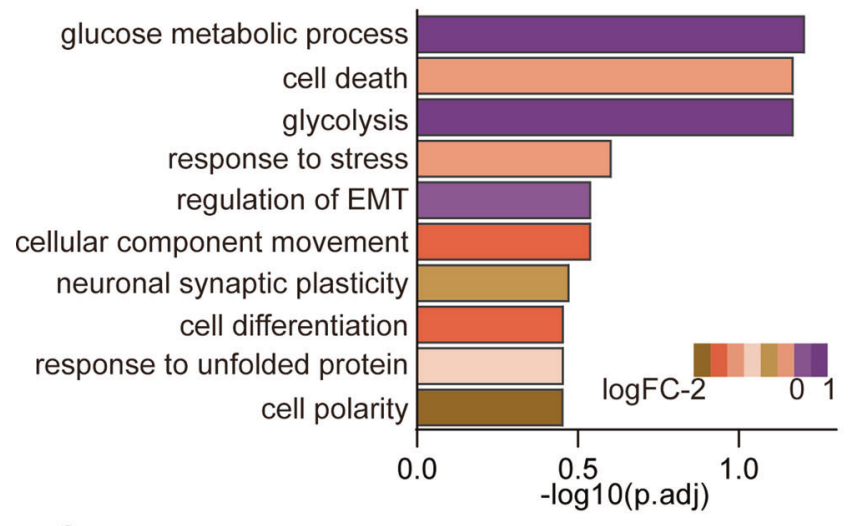

B

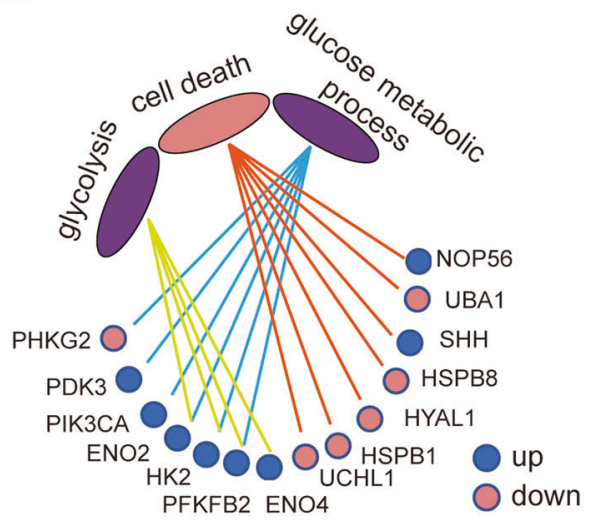

D

C
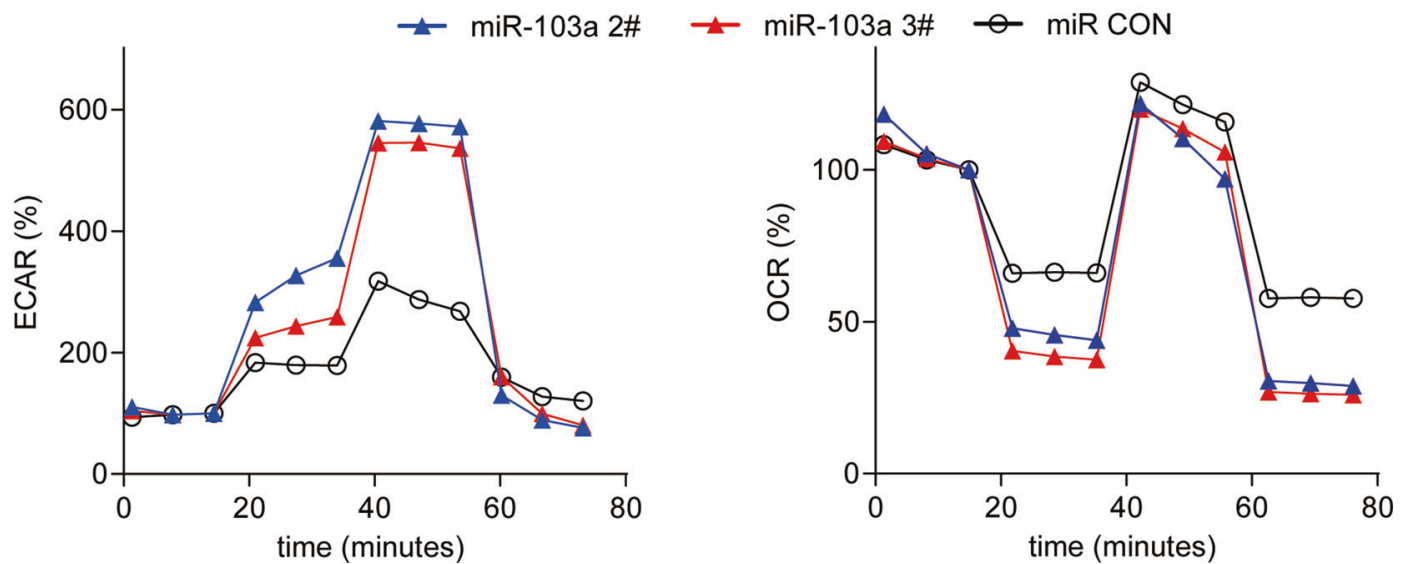

Fig. 5 miR-103a influenced glucose metabolism in HCC cells. A Functional enrichment analysis revealed that miR-103a participated in glucose metabolic processes, cell death and glycolysis biological processes. B Total of 14 genes were matched with these biological functions. C, D, ECAR and OCR of HCC cells were analysed by a Seahorse XFe 96 Extracellular Flux Analyzer. All results are representative of at least three independent experiments.

membrane, and the output was a unitless parameter (cell index). For proliferation assays, $1 \times 10^{4}$ to $3 \times 10^{4}$ cells were seeded into E-plate 16 (ACEA Biosciences) with $200 \mu \mathrm{L}$ DMEM containing $10 \%$ FBS $(n=3)$. The cell index was normalized to the baseline reading at time point 0 and measured every $30 \mathrm{~min}$ for $72 \mathrm{~h}$. Migration and invasion assays were performed in 16well CIM plates (ACEA Biosciences). For migration assays, $1.5 \times 10^{5}$ cells were seeded in triplicate in the upper chamber in a serum-free medium. The upper chamber was then placed on the lower part of the CIM device, which contained DMEM with $10 \%$ FBS as a chemoattractant. The cell index was measured every $30 \mathrm{~min}$ for $48 \mathrm{~h}$. For the invasion assays, the upper chamber of the CIM-16 plate was initially coated with Matrigel (BD Biosciences, Bedford, MA, USA) diluted in serum-free medium at a ratio of 1:20. The next steps were consistent with those for the migration assay.

For the colony formation assay, $8 \times 10^{2}$ cells were seeded in six-well plates and maintained in DMEM with 5\% FBS. After 14 days, the cells were washed with PBS and stained with $0.5 \%$ crystal violet (Sigma, USA).

\section{ECAR and OCR}

A Seahorse XF96 Flux Analyzer (Seahorse Bioscience, Billerica, Massachusetts, USA) was used to measure the oxygen consumption rate (OCR) and extracellular acidification rate (ECAR) in lung cancer cells according to the manufacturer's instructions. Approximately $1 \times 10^{5}$ SMMC-7721 cells per well were seeded into an XF96-well plate and attached overnight. For the assessment of ECAR, cells were incubated with nonbuffered RPMI 1640 under basal conditions, followed by a sequential injection of $10 \mathrm{mM}$ glucose, $1 \mathrm{mM}$ mitochondrial poison (oligomycin, Sigma-Aldrich, Saint Louis, Missouri, USA) and $80 \mathrm{mM}$ glycolysis inhibitor (2-deoxyglucose, 2DG, Sigma-Aldrich). OCR was assessed under basal conditions and after sequential injection of $1 \mu \mathrm{M}$ oligomycin, $1 \mu \mathrm{M}$ fluoro-carbonyl cyanide phenylhydrazone (FCCP) and $2 \mathrm{mM}$ antimycin A and rotenone (SigmaAldrich, Saint Louis, Missouri, USA). Both the ECAR and OCR measurements were normalized to the total protein content.

\section{Target gene prediction of miR-103a and functional enrichment analysis}

The bioinformatic online database miRDB was used to predict potential target genes of miR-103a. The consensus results of the miRDB database and RNA-seq were selected for further analysis. Total RNA was isolated from MHCC97L cells using TRIzol reagent (Invitrogen) and purified by an RNeasy Mini Kit (Qiagen). Transcriptome sequencing was conducted using Illumina HiSeq 2000 by BerryGenomics (Beijing Biotech, China). Gene functional enrichment analyses were performed using EGSEA r-package software, which is a widely used tool for gene functional enrichment. To construct the interaction network, protein-protein interaction data from the STRING database were used. All procedures were conducted according to official protocols and default parameters.

\section{Transfection of microRNA agomiR and antagomiR}

An agomiR is a type of specially labelled and chemically modified doublestranded microRNA that can regulate the biological function of a target gene by mimicking endogenous microRNA. An antagomiR is a type of specifically labelled and chemically modified single-stranded microRNA designed based on the mature microRNA sequence that can inhibit the expression of endogenous microRNA. AgomiR-103a, antagomiR-103a and their respective control materials were procured from RiboBio (Guangzhou, China) and transfected into HCCS and HEK293T cells according to the manufacturer's protocol. The medium was changed once after $24 \mathrm{~h}$ of transfection. 
A

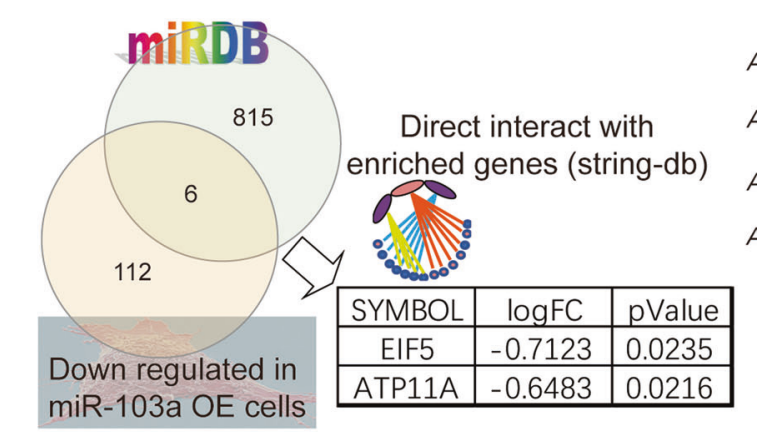

ATP11A (3'UTR, 511-530\#1): 5'-GGATGCCACATGCTGCTGTT-3' ATP11A (3'UTR, 2798-2817\#2): 5'-TCTTATTTGTTGCTGCACAG-3' ATP11A (3'UTR, 2912-2931\#3): 5'-ACAGTGGGCCTGCTGCATGA-3' ATP11A (3'UTR, 2971-2990\#4): 5'-ACAGTGGGCCTGCTGCATGA-3' ATP11A (3'UTR, 3030-3049\#5): 5'-ACAGTGGGCCTGCTGCATGC-3'

EIF5 (3'UTR, 22-41\#1): 5'-TAACAGTATAATGCTGCAA-3'

EIF5 (3'UTR, 3546-3565\#2): 5'-GGAAGCCTAATGCTGCAGTC-3' |||||| $\mid$ miR-103a: 3'-AGUAUCGGGACAUGUUACGACGA-5'

C
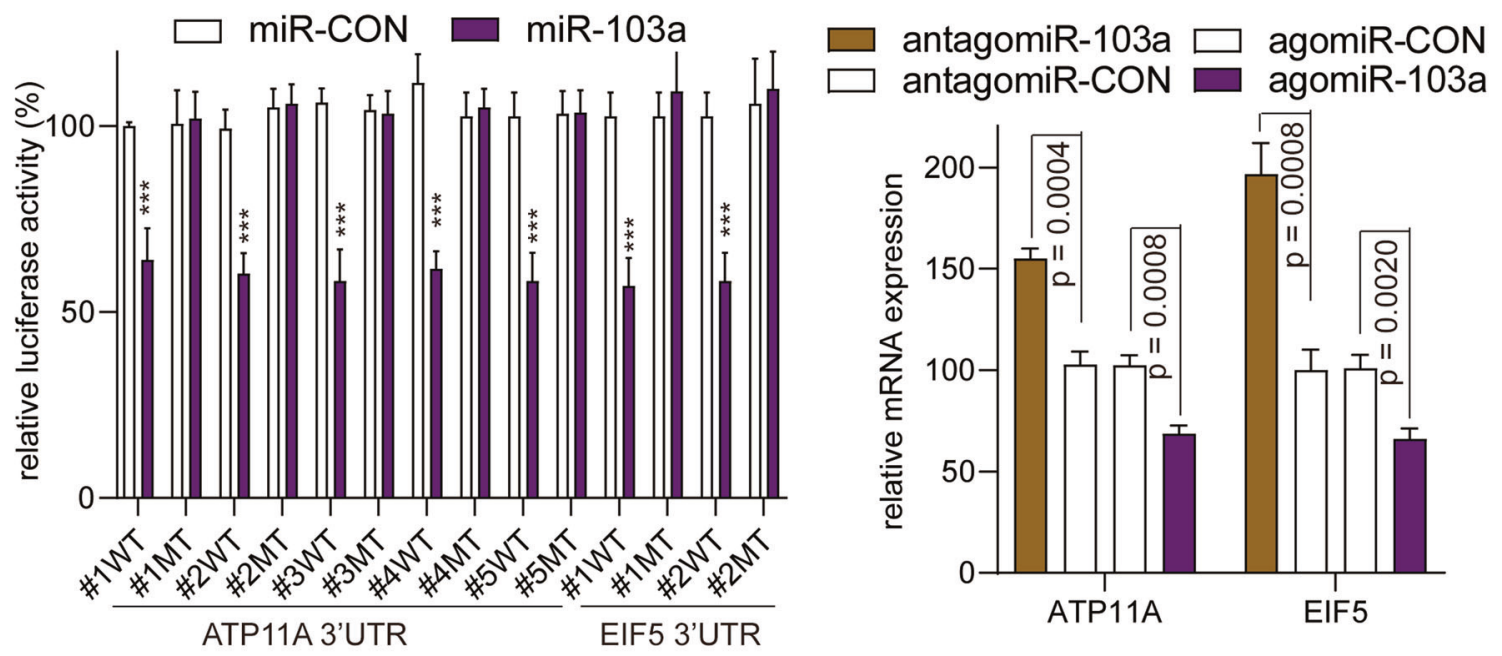

$E$
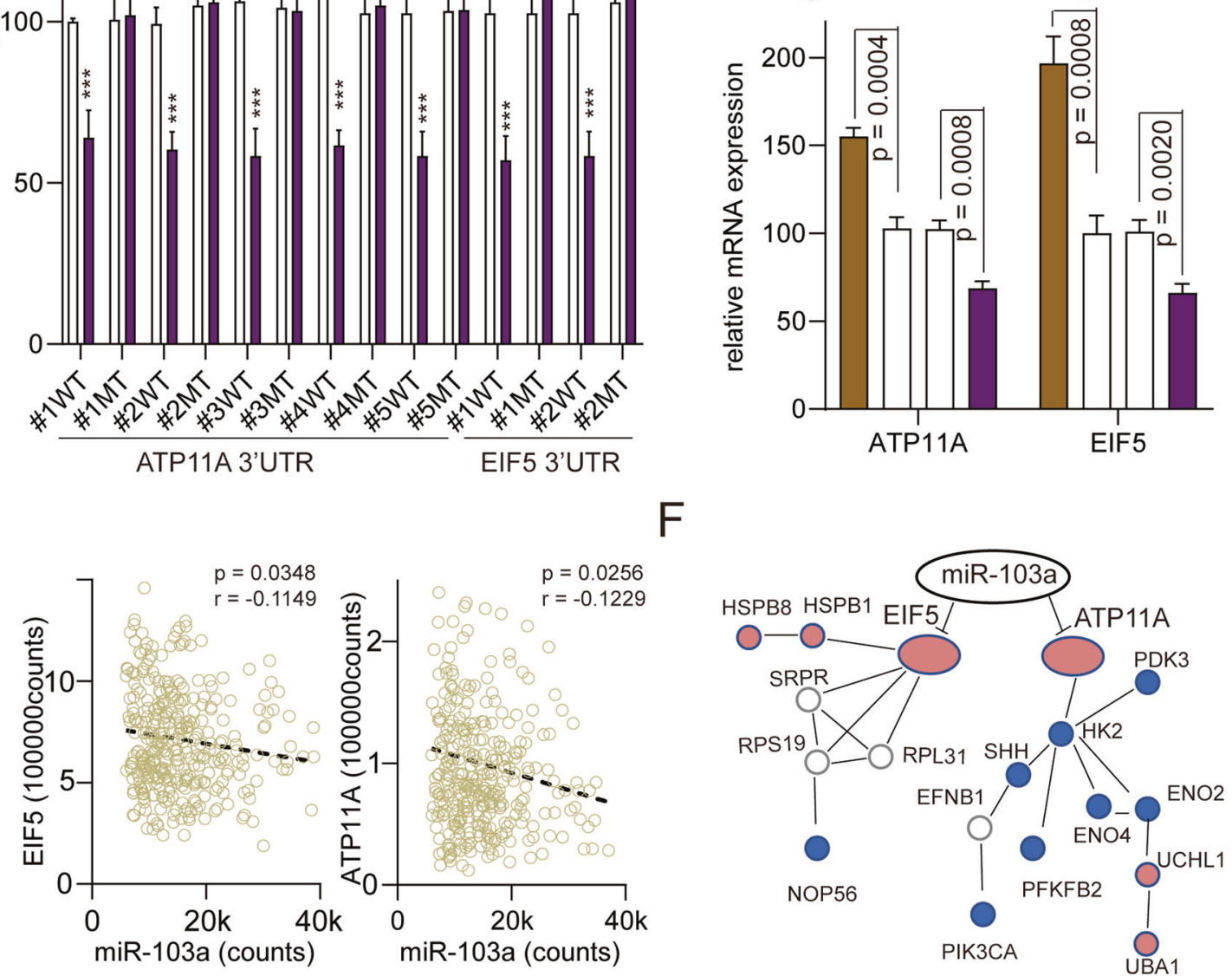

Fig. 6 ATP11A and EIF5 were direct targets of miR-103a. A Identification of potential miR-103a target genes by microRNA prediction databases and downregulated genes in miR-103a-overexpressing HCC cells. B Predicted miR-103a target sequences in the 3'-UTR of ATP11A and EIF5. In addition, the target sequences were mutated. C Luciferase reporter activity of WT or MT ATP11A and EIF5 promoters in SMMC7721 cells transfected with miR-103a mimics and related constructs. D MRNA levels of ATP11A and EIF5 in SMMC-7721 cells treated with miR103a agomiRs or antagomiRs. E Correlations between the mRNA expression of miR-103a and ATP11A or EIF5 in TCGA datasets analysed by Pearson's correlation analysis. F miR-protein network analysis using STRINGdb showing that ATP11A and EIF5 were glycolysis-associated target genes of miR-103a. ${ }^{* *} p<0.01,{ }^{* * *} p<0.001$. All results are representative of at least three independent experiments.

\section{Luciferase reporter assay}

The ATP11A/EIF5 $3^{\prime}$-UTR (untranslated region) and mutants were obtained from RiboBio (Guangzhou, China). HEK293T cells were cotransfected with pmiR-103a agomiR or negative controls (NCs). Luciferase activity was measured $24 \mathrm{~h}$ after transfection using the Dual$\mathrm{Glo}^{\circledR}$ Luciferase Assay System (Promega) according to the manufacturer's instructions.

\section{Tumour xenograft models}

A subcutaneous xenograft mouse model was used to assess tumour growth. Animal experiments were approved by the Ethics Committee of Renji Hospital, Shanghai Jiao Tong University School of Medicine. Female nude mice (age, 4-5 weeks; weight, 15-20 g; Institute of Zoology, Chinese Academy of Sciences) were randomly divided into two groups: the miR103a overexpression group and the CON group (at least 6 per group). 
A

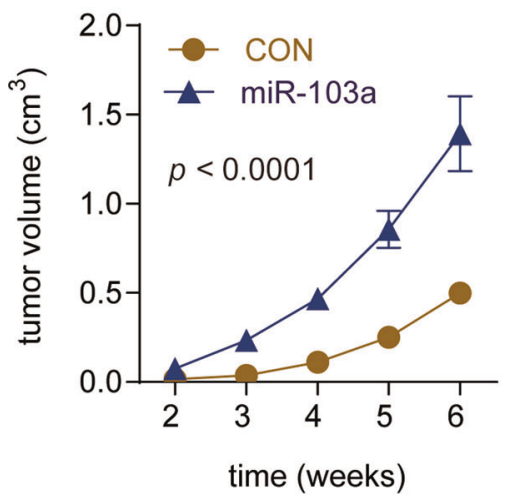

D

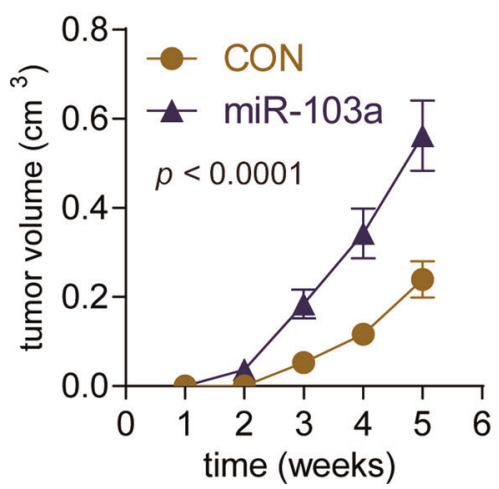

B

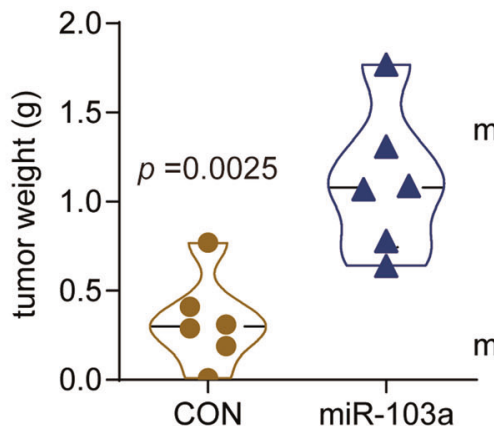

E

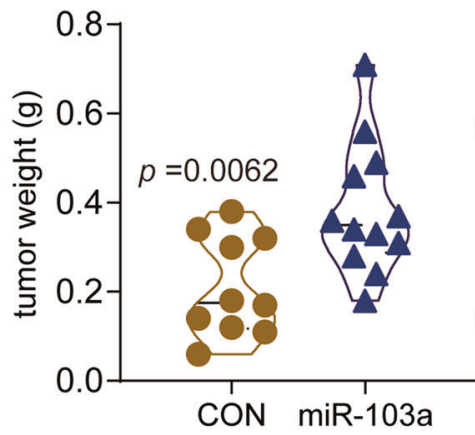

C

miR-103a

CON

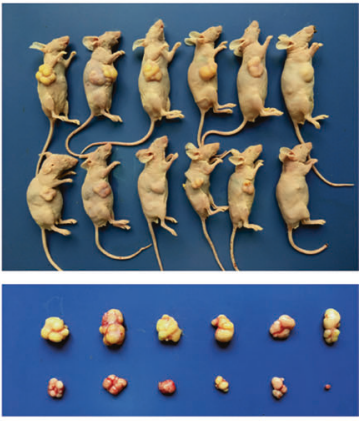

$\mathrm{F}$

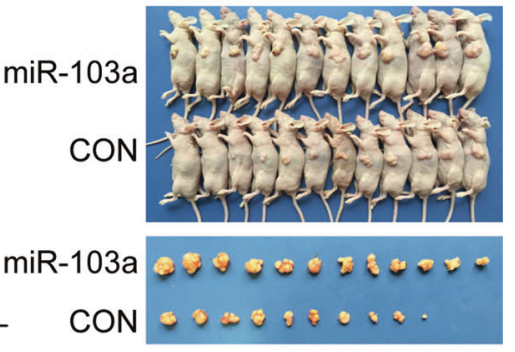

Fig. 7 miR-103a promoted cell growth in vivo. miR-103a overexpression promoted SMMC-7721/MHCC97L cell growth in vivo. A, D Significantly higher tumour volumes were observed in mouse xenografts with HCC cells transfected with the miR-103a mimic than in control xenografts. B, E Tumour weights were significantly higher in mouse xenografts. C, F Representative images of xenograft tumours are shown. ${ }^{* *} p<0.01,{ }^{* * *} p<0.001$.

A total of $2 \times 10^{6}$ SMMC-7721 cells in $100 \mu \mathrm{L}$ of DMED without FBS were injected into nude mice. A total of $2 \times 10^{6}$ MHCC97L cells in $100 \mu \mathrm{L}$ of DMED without FBS were injected into nude mice. The tumour volume was measured by calliper measurements every week and calculated with the formula (length $\times$ width $\left.^{2}\right) / 2$.

\section{Statistical analysis}

Data are expressed as the mean \pm standard deviation (SD) from at least three independent experiments. Statistical differences between groups were evaluated by Student's $t$ test (paired/unpaired). Two-way analysis of variance (ANOVA) followed by Tukey's multiple comparisons test was performed to compare significant differences and calculate the $P$-value between the different groups. Pearson correlation tests were performed for the correlation analyses. A survival analysis was performed with the Kaplan-Meier method, and the log-rank test was used for comparisons. Statistical results were analysed using Prism software (GraphPad Software). A probability of 0.05 or less was considered statistically significant.

\section{REFERENCES}

1. Miller KD, Nogueira L, Mariotto AB, Rowland JH, Yabroff KR, Alfano CM, et al. Cancer treatment and survivorship statistics, 2019. CA. 2019;69:363-85.

2. Hu W, Zheng S, Guo H, Dai B, Ni J, Shi Y, et al. PLAGL2-EGFR-HIF-1/2alpha signaling loop promotes HCC progression and erlotinib insensitivity. Hepatology. 2021;73:674-91.

3. Dai $H$, Jia G, Wang $H$, Yang J, Jiang $H$, Chu M. Epidermal growth factor receptor transactivation is involved in the induction of human hepatoma SMMC7721 cell proliferation by insufficient radiofrequency ablation. Oncol. Lett. 2017;14:2463-7.

4. Siegel RL, Miller KD, Jemal A. Cancer statistics, 2019. CA. 2019;69:7-34.

5. de Martel C, Ferlay J, Franceschi S, Vignat J, Bray F, Forman D, et al. Global burden of cancers attributable to infections in 2008: a review and synthetic analysis. Lancet Oncol. 2012;13:607-15.
6. Cao MQ, You AB, Zhu XD, Zhang W, Zhang YY, Zhang SZ, et al. miR-182-5p promotes hepatocellular carcinoma progression by repressing FOXO3a. J. Hematol. Oncol. 2018;11:12.

7. Ma XL, Shen MN, Hu B, Wang BL, Yang WJ, Lv LH, et al. CD73 promotes hepatocellular carcinoma progression and metastasis via activating PI3K/AKT signaling by inducing Rap1-mediated membrane localization of P110beta and predicts poor prognosis. J. Hematol. Oncol. 2019;12:37.

8. Ambros V. The functions of animal microRNAs. Nature. 2004;431:350-5.

9. Bartel DP. MicroRNAs: genomics, biogenesis, mechanism, and function. Cell. 2004;116:281-97.

10. Fabian MR, Sonenberg N, Filipowicz W. Regulation of mRNA translation and stability by microRNAs. Annu. Rev. Biochem. 2010;79:351-79.

11. Bartel DP. MicroRNAs: target recognition and regulatory functions. Cell. 2009;136:215-33.

12. Prodromidou K, Vlachos IS, Gaitanou M, Kouroupi G, Hatzigeorgiou AG, Matsas R. MicroRNA-934 is a novel primate-specific small non-coding RNA with neurogenic function during early development. eLife. 2020;9:e50561.

13. Meerson A, Traurig M, Ossowski V, Fleming JM, Mullins M, Baier LJ. Human adipose microRNA-221 is upregulated in obesity and affects fat metabolism downstream of leptin and TNF-alpha. Diabetologia. 2013;56:1971-9.

14. Arderiu G, Peña E, Aledo R, Juan-Babot O, Crespo J, Vilahur G, et al. MicroRNA-145 regulates the differentiation of adipose stem cells toward microvascular endothelial cells and promotes angiogenesis. Circulation Res. 2019;125:74-89.

15. He T, Shen H, Wang S, Wang Y, He Z, Zhu L, et al. MicroRNA-3613-5p promotes lung adenocarcinoma cell proliferation through a RELA and AKT/MAPK positive feedback loop. Mol. Ther. Nucleic Acids. 2020;22:572-83.

16. Xiao $Y$, Sun $Y, M a X$, Wang $C$, Zhang $L$, Wang J, et al. MicroRNA-22 inhibits the apoptosis of vascular smooth muscle cell by targeting p38MAPKalpha in vascular remodeling of aortic dissection. Mol. Ther. Nucleic Acids. 2020;22:1051-62.

17. Dhingra R, Lin J, Kirshenbaum LA. Disruption of RIP1-FADD complexes by MicroRNA-103/107 provokes necrotic cardiac cell death. Circulation Res. 2015;117:314-6.

18. Wang $X$, He Y, Mackowiak B, Gao B. MicroRNAs as regulators, biomarkers and therapeutic targets in liver diseases. Gut. 2021;70:784-95. 
19. Wei X, Zhao L, Ren R, Ji F, Xue S, Zhang J, et al. MiR-125b loss activated HIF1alpha/ pAKT loop, leading to transarterial chemoembolization resistance in hepatocellular carcinoma. Hepatology. 2021;73:1381-98.

20. Komoll RM, Hu Q, Olarewaju O, von Döhlen L, Yuan Q, Xie Y, et al. MicroRNA-342$3 p$ is a potent tumour suppressor in hepatocellular carcinoma. J. Hepatol. 2021;74:122-34.

21. Wu H, Zhang W, Wu Z, Liu Y, Shi Y, Gong J, et al. miR-29c-3p regulates DNMT3B and LATS1 methylation to inhibit tumor progression in hepatocellular carcinoma. Cell Death Dis. 2019;10:48.

22. Xu WP, Liu JP, Feng JF, Zhu CP, Yang Y, Zhou WP, et al. miR-541 potentiates the response of human hepatocellular carcinoma to sorafenib treatment by inhibiting autophagy. Gut. 2020;69:1309-21.

23. Chen L, Liu D, Yi X, Qi L, Tian X, Sun B, et al. The novel miR-1269b-regulated protein SVEP1 induces hepatocellular carcinoma proliferation and metastasis likely through the PI3K/Akt pathway. Cell death Dis. 2020;11:320.

24. Li S, Shao J, Lou G, Wu C, Liu Y, Zheng M. MiR-144-3p-mediated dysregulation of EIF4G2 contributes to the development of hepatocellular carcinoma through the ERK pathway. J Exp Clin Cancer Res. 2021;40:53.

25. Liu Y, Lu LL, Wen D, Liu DL, Dong LL, Gao DM, et al. MiR-612 regulates invadopodia of hepatocellular carcinoma by HADHA-mediated lipid reprogramming. J Hematol Oncol. 2020;13:12.

26. Fang F, Chang RM, Yu L, Lei X, Xiao S, Yang H, et al. MicroRNA-188-5p suppresses tumor cell proliferation and metastasis by directly targeting FGF5 in hepatocellular carcinoma. J. Hepatol. 2015;63:874-85.

27. Kudo Y, Sugimoto M, Arias E, Kasashima H, Cordes T, Linares JF, et al. PKClambda/ iota loss induces autophagy, oxidative phosphorylation, and NRF2 to promote liver cancer progression. Cancer Cell. 2020;38:247-62. e11.

28. Xu D, Wang Z, Xia Y, Shao F, Xia W, Wei Y, et al. The gluconeogenic enzyme PCK1 phosphorylates INSIG1/2 for lipogenesis. Nature. 2020;580:530-5.

29. Trajkovski M, Hausser J, Soutschek J, Bhat B, Akin A, Zavolan M, et al. MicroRNAs 103 and 107 regulate insulin sensitivity. Nature. 2011;474:649-53.

30. Martello G, Rosato A, Ferrari F, Manfrin A, Cordenonsi M, Dupont S, et al. A MicroRNA targeting dicer for metastasis control. Cell. 2010;141:1195-207.

31. Chen HY, Lin YM, Chung HC, Lang YD, Lin CJ, Huang J, et al. miR-103/107 promote metastasis of colorectal cancer by targeting the metastasis suppressors DAPK and KLF4. Cancer Res. 2012;72:3631-41.

32. Zheng J, Liu Y, Qiao Y, Zhang L, Lu S. miR-103 promotes proliferation and metastasis by targeting KLF4 in gastric cancer. Int J Mol Sci. 2017;18:1-13.

33. Xiong $B$, Lei $X$, Zhang $L$, Fu J. miR-103 regulates triple negative breast cancer cells migration and invasion through targeting olfactomedin 4. Biomed Pharmacother. $=$ Biomedecine pharmacotherapie. 2017;89:1401-8.

34. Yang D, Wang JJ, Li JS, Xu QY. miR-103 functions as a tumor suppressor by directly targeting programmed cell death 10 in NSCLC. Oncol. Res. 2018;26:519-28.

35. Wang DS, Zhong B, Zhang MS, Gao Y. Upregulation of serum miR-103 predicts unfavorable prognosis in patients with colorectal cancer. Eur Rev Med Pharmacol Sci. 2018;22:4518-23.

36. Tan Y, Zhao L. miR-103 promotes hepatocellular carcinoma cell proliferation and migration in the simulation transition zone of RFA through PI3K/Akt signaling pathway by targeting PTEN. Int J Clin Exp Pathol. 2020;13:473-9.

37. Han LL, Yin XR, Zhang SQ. miR-103 promotes the metastasis and EMT of hepatocellular carcinoma by directly inhibiting LATS2. Int J Oncol. 2018;53:2433-44.

38. Xia W, Ni J, Zhuang J, Qian L, Wang P, Wang J. MiR-103 regulates hepatocellular carcinoma growth by targeting AKAP12. Int J Biochem Cell Biol. 2016;71:1-11.

39. Fang JH, Zhang ZJ, Shang LR, Luo YW, Lin YF, Yuan Y, et al. Hepatoma cellsecreted exosomal microRNA-103 increases vascular permeability and promotes metastasis by targeting junction proteins. Hepatology. 2018;68:1459-75.

40. Zhang L, Yao W, Xia J, Wang T, Huang F. Glucagon-induced acetylation of energysensing factors in control of hepatic metabolism. Int J Mol Sci. 2019;20:1-20.

41. Cohen JC, Horton JD, Hobbs HH. Human fatty liver disease: old questions and new insights. Science. 2011;332:1519-23.
42. Hoxhaj G, Manning BD. The PI3K-AKT network at the interface of oncogenic signalling and cancer metabolism. Nat Rev Cancer. 2020;20:74-88.

43. Ganapathy-Kanniappan S, Geschwind JF. Tumor glycolysis as a target for cancer therapy: progress and prospects. Mol Cancer. 2013;12:152

44. Vander Heiden MG, Cantley LC, Thompson CB. Understanding the Warburg effect: the metabolic requirements of cell proliferation. Science. 2009;324:1029-33.

\section{AUTHOR CONTRIBUTIONS}

Y.W. and X.L. designed the experiments, revised the manuscript, and supervised the progress throughout this study. Y.L, Y.Z., B.X. and N.T. carried out the experiments and wrote the manuscript and analysed the data. J.H., S.L., Y.P., H.X., J.A., J.Y., X.L. and L.W. participated in the experiments. All authors reviewed and approved the final manuscript.

\section{FUNDING}

This study was supported by grants from the National Natural Science Foundation of China (No. 81572454 and 81773173) and the Research Project of Science and Technology Development Fund of Shanghai Pudong New Area (PKJ2019-Y30).Ethics All clinical studies were approved by the ethics committees of the Shanghai Cancer Institute. All animal experimental procedures acquired official approval of the Institutional Animal Care and Use Committee of Model Animal Research Center of Shanghai Cancer Institute and the Institutional Animal Care and Use Committee of Shanghai Institute of Materia Medica, Chinese Academy of Sciences.

\section{CONFLICT OF INTEREST}

The authors declare no competing interests.

\section{ADDITIONAL INFORMATION}

Supplementary information The online version contains supplementary material available at https://doi.org/10.1038/s41419-021-03905-3.

Correspondence and requests for materials should be addressed to Y.W. or X.L.

Reprints and permission information is available at http://www.nature.com/ reprints

Publisher's note Springer Nature remains neutral with regard to jurisdictional claims in published maps and institutional affiliations.

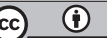

Open Access This article is licensed under a Creative Commons Attribution 4.0 International License, which permits use, sharing, adaptation, distribution and reproduction in any medium or format, as long as you give appropriate credit to the original author(s) and the source, provide a link to the Creative Commons license, and indicate if changes were made. The images or other third party material in this article are included in the article's Creative Commons license, unless indicated otherwise in a credit line to the material. If material is not included in the article's Creative Commons license and your intended use is not permitted by statutory regulation or exceeds the permitted use, you will need to obtain permission directly from the copyright holder. To view a copy of this license, visit http://creativecommons. org/licenses/by/4.0/.

(c) The Author(s) 2021 